\title{
Plexiform or multinodular schwannoma of posterior tibial nerve: case report with review of literature.
}

\author{
Prahalad Kumar Singhi*, Sivakumar Raju, Somashekar V., Bharat Kumar, Anil Kumar
}

Department of Orthopaedics, Preethi Hospitals Pvt. Ltd., Madurai, Tamilnadu, India

Received: 24 June 2020

Accepted: 28 July 2020

\section{*Correspondence:}

Dr. Prahalad Kumar Singhi,

E-mail: docpsin2001@yahoo.co.in

Copyright: ( $)$ the author(s), publisher and licensee Medip Academy. This is an open-access article distributed under the terms of the Creative Commons Attribution Non-Commercial License, which permits unrestricted non-commercial use, distribution, and reproduction in any medium, provided the original work is properly cited.

\begin{abstract}
A schwannoma is a benign tumor that develops from the Schwann cells, which assists conduction of nerve impulses and located in the nerve sheath of peripheral or cranial nerves. Plexiform or multinodular Schwannoma of posterior tibial nerve is a rare presentation, can cause diagnostic dilemma with Lumbosacral radiculopathy, Tarsal tunnel syndrome, Entrapment neuropathy or Chronic regional pain syndrome. Unexplained leg pain, a positive Tinel's sign with or without a palpable swelling and Magnetic Resonance Imaging will clinch the diagnosis after excluding other causes. In symptomatic cases with long standing complaints, surgical resection yields satisfactory outcome. We present an interesting case of plexiform schawannoma along posterior tibial nerve with review of literature.
\end{abstract}

Keywords: Plexiform, Multinodular, Schwannoma, Posterior tibial nerve

\section{INTRODUCTION}

A schwannoma is a benign tumor that develops from the Schwann cells, which assists conduction of nerve impulses located in the nerve sheath of peripheral or cranial nerves. Also known as neurilemomas or neuromas. Malignant transformation is less frequent and referred as a soft tissue sarcoma. Often these tumours seen in trunk, head and neck region along brachial plexus and less often being described in upper and lower limbs. ${ }^{1}$ Often many swellings can occur along the peripheral nerves with or without other features of neurofibromatosis.

Benign peripheral nerve sheath tumors can be divided into 2 groups: schwannoma and neurofibroma. Both contain cells that are closely related to normal schwann cells. Schwannomas are, overall, less common than neurofibromas. ${ }^{2}$ Schwanomma of peripheral nerves are asymptomatic and this is attributed for the delayed clinical diagnosis. ${ }^{3}$ Plexiform or multinodular schwa- nomma is a less common variant. The symptoms are due to compressive radiculopathy or mass effect. Published literature has described these lesions as solitary and occurs along the posterior tibial nerve in lower limbs. We report a rare case example of multinodular or plexiform schwannoma along posterior tibial nerve occurring as multiple string of beads appearance.

\section{CASE REPORT}

A thirty five years old male presented to our hospital with complains of radiating pain along the left lower leg, ankle and sole of foot for past three years and six month. Patient had noticed painful local swelling on inner aspect of ankle for past year. He doesn't have any weakness of lower limbs or difficulty in wearing shoe wear. Earlier he was treated for Sciatica with Drugs, Physiotherapy and lifestyle modifications which were revealed by his earlier Radiology report of Lumbar spine and prescriptions. He was not relieved of his symptoms. 
On clinical examination there was localised swelling approximately of size $4 \times 3 \times 3 \mathrm{~cm}$ at medial aspect left ankle just behind the medial malleolus. Swelling was exquisitely tender, firm and non-pulsatile. It was not fixed to underlying bone or overlying skin and was freely mobile side to side but not longitudinally. Movements of the ankle were free and there was no distal neuro-vascular deficit, however Tinel's sign was distinctly positive. Examination of the lower extremity and lower back was inconclusive. Blood work up and radiology of the lumbosacral spine was done previously which was normal. MRI of the left ankle revealed multiple ovoid shaped lesions with string of bead appearance seen along the course of posterior tibial nerve behind the tibia and extending into tarsal tunnel (Figure 1).

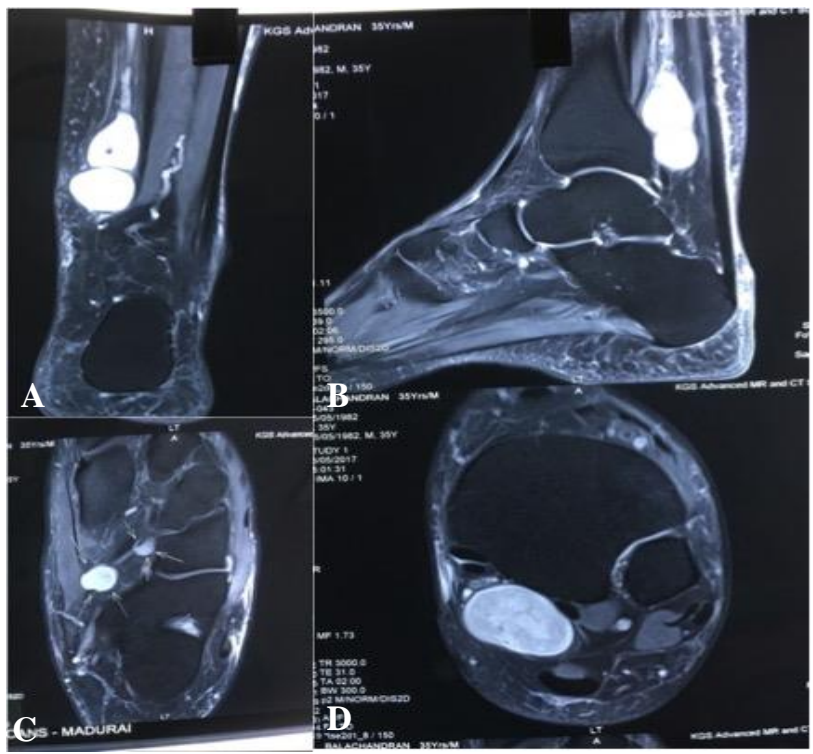

Figure 1: Magnetic Resonance Imaging of the multinodular tumour with one extending in tarsal tunnel. A) Upper lesion is measuring $2.70 \times 1.50 \mathrm{~cm}, \mathrm{~B}$ )

Lower lesion is measuring $1.98 \times 1.93 \mathrm{~cm} \mathrm{C}$ ) Third small satellite nodule seen adjacent to tibialis posterior insertion site measuring $0.91 \times 0.86 \mathrm{~cm} D$ ) The last small nodule is measuring $0.70 \times 0.67 \mathrm{~cm}$.

Based on clinical and radiological examination a diagnosis of schwannoma of the tibial nerve was made and was advised surgical resection. Under regional anaesthesia after preparation an direct incision was taken over the swelling. Soft tissue plains were dissected. There were multiple well-defined fusiform swellings noted along the posterior tibial nerve. Adequate exposure of the nerve proximally and distally to the tumour was done. The swelling was opened by a longitudinal incision and a plane was developed between the nerve bundles the entry and exit points identified and then removed in toto (Figure 2). Small fascicles entering the tumour also required removal. The continuity of the nerve fibres of the main trunk was ensured. There were three masses which were well circumscribed, encapsulated, firm and gray in colour with areas of cystic change, fourth was a tiny mass in tarsal tunnel. The largest one was $2.5 \times 1.5$ $\mathrm{cm}$ in size. Histopathological examination showed areas comprising of hyper and hypocellular zones i.e. Antoni A and Antoni B pattern. In the hypercellular areas, there was presence of nuclear palisading and Verocay bodies. The cells were spindle shaped with fibrillary eosinophillic cytoplasm, round to oval nuclei and inconspicuous nucleoli confirming the diagnosis of schwannoma (Figure 3). Post-operative period was uneventful with complete healing, no deficit and non recurrence at last follow up of 3 years.

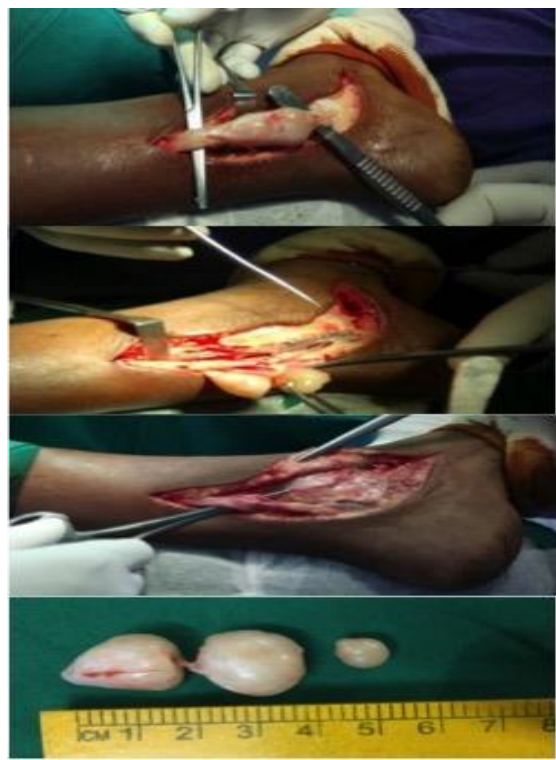

Figure 2: Surgical steps of complete excision the multinodular tumour.

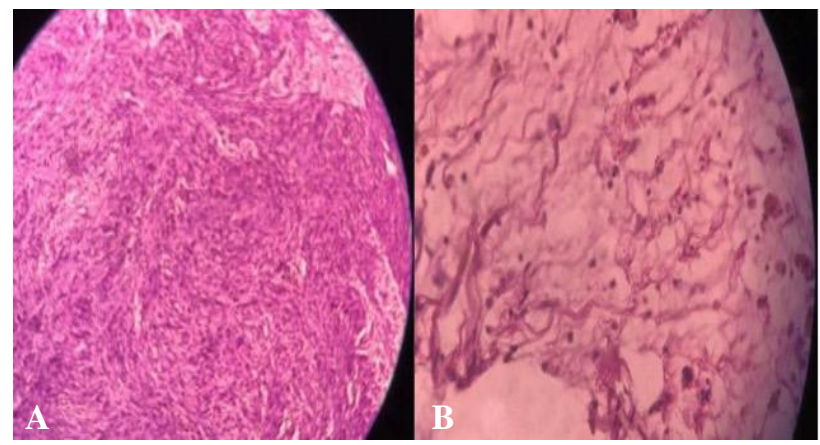

Figure 3: Histopathological slide showing Antoni A and Antoni B pattern. A) Hypercellular zone B) Hypocellular zone.

\section{DISCUSSION}

Schwannoma, neurilemmoma, or Schwann cell tumour is well-defined solitary nerve sheath tumor accounting for $5 \%$ of soft tissue tumors, and are benign most often [3]. Schwannomas can occur at any age usually peaks in fourth decade of age and the incidence is same in both genders with no difference based on ethnic or racial backgrounds. Schnnomma can be genetically transferred from parents to children if one of the parents is affected. ${ }^{4}$ 
Knight et al reviewed 234 cases, 64 occurred in the pelvis and lower limb. Tibial, sciatic and common peroneal nerve is commonly affected few cases occur in plantar branches of tibial nerve causing tarsal tunnel syndrome or heel pain. ${ }^{5}$

Schwannomas can occur as eccentric slow growing mass along the peripheral nerves arising from their sheath. Plexiform schwannoma is a rare variant with a plexiform or multinodular growth pattern, which is represented in our case. The presenting symptoms can be basically slow growing mass with delayed appearance, numbness, rarely muscle weakness, pinning and needling sensation, night aching, neurogenic, burning or sharp pains. Painless mass with associated neuropathic pain peripherally leads diagnostic dilemma. ${ }^{6}$ These patients are often misdiagnosed as lumbosacral radiculopathy, tarsal tunnel syndrome, entrapment neuropathy and even chronic regional pain syndrome. Differential diagnosis will be swelling arising from adjacent structures like blood vessel like arterial aneurysm, deep venous thrombosis or varicosities and tendon sheath tumors like giant cell tumor, tenosynovitis, and ganglion cyst. Other possibilities include neurofibroma, neurofibrosarcoma, leiomyoma, histiocytoma, desmoid tumor, pigmented villonodular synovitis, tarsal tunnel syndrome, and neuroma. . $^{2,3,4,5}$ Our patient was earlier diagnosed as lumbar radiculopathy for more than three years before he presented to us. Many authors have reported cases where the diagnosis was delayed for a very long time. ${ }^{7}$ When the fascicles are entrapped proximally the palpation of nerve often elicits characteristic Tinels sign, which could be used as diagnostic tool. ${ }^{1}$

Ultrasound and Magnetic Resonance Imaging confirms the diagnosis, a well-circumscribed, encapsulated mass with a heterogeneous signal infiltering the nerve, exact location of the nerve entering or exiting the mass can well be assessed. The split fat sign, fascicular sign, and target sign are some signs described on MRI in radiology data. The fascicular sign is the appearance of the fascicular bundles in neurogenic tumors and normal nerves. The target sign has been described as having a biphasic pattern on T2-weighted images of schwannomas (sometimes in neurofibroma), characterized by central decreased signal intensity secondary to fibrous tissue and peripheral increased signal intensity secondary to myxomatous elements. The split-fat sign indicates a rim of fat surrounding the tumor, which originates from the nerve in an intermuscular location. ${ }^{8,4}$

Even though these tumors are benign when symptomatic, complete excision is the treatment of choice. Surgical resection is relatively easy due its eccentricity, after isolating the mass a longitudinal incision is made on the capsule the entry and exit points of the nerve are identified and excised to remove the tumor in toto. However reconstructive microsurgical skills may be necessary if there is penetration of nerve or accidental iatrogenic injury if nerve loss is less than $3 \mathrm{~cm}$ nerve repair using autograft or syntheic graft has been reported. ${ }^{9}$ Small benign schwannomas may be just monitored. Radiation may be used in some cases of inaccessible regions or in recurrence. Malignant schwannomas need in depth analysis requiring chemo-therapy, immunotherapy or both. If schwannomas develops on a smaller nerve, it may difficult to separate the tumor from the nerve and one has to sacrifice it. Recurrence may be noted if not removed completely. ${ }^{10,11}$

In our case there were multiple swellings along the nerve, requiring careful dissection and in toto excision. Post excision diagnosis of schwannoma was confirmed with histopathological examination along with immune histochemical markers like S100, Factor XIIIa, CD56, Ki67, neurofilament protein, CD34, and calretinin. Immunohistochemistry can differentiate between schwannomma and neurofibromatosis. Immunohistochemical marker calretinin is specific for schwannomas and CD56 was sensitive to it differentiating with, a high CD34 expression for neurofibromas. ${ }^{12,13}$ The prognosis of schwannoma after excision is relatively favourable. Eccentricity in its location to the nerve and careful dissection avoids any neurological deficit. Minimal sensory or functional impairment can occur which will recover with time has been reported. The reason for impairment could be tumour infiltrating fascicle, irreversible compressive neuropathy or iatrogenic injury. Careful microsurgical dissection is of paramount importance and surgical repair of nerve is necessary in case of intra operative injury. ${ }^{10}$ In our case surgical resection was challenging because of multinodular masses, which is less common. Post-surgery there was no added neurological deficit noted resulting in good outcome.

\section{CONCLUSION}

Schwannomma are benign peripheral nerve sheath tumours, slow growing so missed most often or have delayed presentation. Plexiform or multinodular Schwannoma of posterior tibial nerve is a rare presentation can cause diagnostic dilemma. Ultrasound and MRI is key in clinching the diagnosis. In symptom-matic cases surgical resection yields satisfactory out-come.

\section{Funding: No funding sources \\ Conflict of interest: None declared \\ Ethical approval: Not required}

\section{REFERENCES}

1. Birch R, Bonney G, Wynn Parry CB. The peripheral nervous system and neoplastic disease. In: Surgical disorders of peripheral nerves. Edinburgh: Churchill Livingstone; 1998: 335-352.

2. Kim DH, Murovich JA, Tiel RL, Moes G, Kline DG. A series of 397 peripheral neural sheath tumors: 30year experience at Louisiana State University Health Sciences Center. J Neurosurg. 2005;102:246-55. 
3. Nawabi D, Sinisi M. Schwannoma of the posterior tibial nerve: the problem of delay in diagnosis. J Bone Joint Surg Br. 2007;89(6):814-6.

4. Albert, P., Patel, J., Badawy, K., Weissinger, W., Brenner, M., Bourhill, I., et al. Peripheral Nerve Schwannoma: A Review of Varying Clinical Presentations and Imaging Findings. The Journal of Foot and Ankle Surgery. 2017;56(3), 632-7.

5. Knight DM, Birch R, Pringle J. Benign solitary schwannomas: a review of 234 cases. J Bone Joint Surg Br. 2007;89:382-7.

6. Kransdorf M. Benign soft-tissue tumors in a large referral population: Distribution of specific diagnosis by age, sex and location. AJR Am J Roentgenol. 1995;164:395-402.

7. White NB. Neurilemomas of the extremities J Bone Joint Surg. 1967;49A:1605-10.

8. Beaman FD, Kransdorf MJ, Menke DM. Schwannoma: radiologic-pathologic correlation. Radiographics. 2004;24:1477-81.

9. Mott RC, Dellon AL. Multiple schwannomas of the foot: case report and strategy for treatment. J Am Podiatr Med Assoc. 2003;93:51-7.
10. Smith W, Amis J. Neurilemmona of the tibial nerve: a case report. J Bone Joint Surg[Am].1992;74-A: 44 3-4.

11. Ghaly R. A posterior tibial nerve neurilemmoma unrecognized for 10 years:case report. Neurosurgery. 2001;48:668-72.

12. Fine SW, McClain SA, Li M: Immunohistochemistry staining for calretinin is useful for differentiating schwannomas from neurofibromas. Am J Clin Pathol. 2004;122:552-9.

13. Park JY, Park H, Park NJ. Use of calretinin, CD56, and CD34 for differential diagnosis of schwannoma and neurofibroma. The Korean Journal of Pathology. 2011;45:30-5.

Cite this article as: Singhi PK, Raju S, Somashekar V, Kumar B, Kumar A. Plexiform or multinodular schwannoma of posterior tibial nerve: case report with review of literature. Int J Res Orthop 2020;6:1135-8. 